\title{
The Ratio of Triglyceride to High-density Lipoprotein Cholesterol as an Indicator of Risk Stratification for Atherosclerotic Cardiovascular Disease in a Clinical Setting
}

\author{
Shigemasa Tani \\ Key words: atherosclerotic cardiovascular disease, coronary artery disease, LDL particle size, TG/HDL-C \\ ratio
}

(Intern Med 59: 2639-2640, 2020)

(DOI: 10.2169/internalmedicine.5344-20)

\begin{abstract}
Large-scale clinical studies conducted to verify the effects of statins, statins plus concomitant ezetimibe therapy (1), and treatment with a protein convertase subtilisin/kexin type 9 (PCSK9) inhibitor designed to prevent the development of coronary artery disease (CAD) (2) have established the usefulness of low-density lipoprotein cholesterol (LDL-C)lowering therapy. In addition, countermeasures against residual independent risk factors, such as hypertriglyceridemia (high TG) and low serum levels of high-density lipoprotein cholesterol (HDL-C) (3), are also emphasized. However, the usefulness of reducing serum TG levels with fibrates or increasing serum HDL-C levels using cholesteryl transfer protein (CETP) inhibitors or niacin for preventing CAD (4) has yet to be demonstrated in a large-scale clinical study.
\end{abstract}

Visceral obesity, a symptom of the disease state of metabolic syndrome, has been seen to lead to a lipid profile characterized by high TG and low HDL-C, the management of which is difficult in clinical settings (3). In addition, it has been reported that the TG/HDL-C ratio is a useful estimator of the LDL particle size, with a higher TG/HDL-C ratio indicating a smaller LDL particle size. In epidemiologic studies, the TG/HDL-C ratio has been recognized as a reliable risk marker of atherosclerotic cardiovascular disease (ASCVD) (5). In demonstrating its usefulness, Moriyama showed that the TG/HDL-C ratio can be easily estimated even in clinical settings using combined serum small-dense LDL (6) and malondialdehyde-modified LDL (7), representing atherogenic LDL subclasses (8). His study also supports the possibility of applying the TG/HDL-C ratio as a new management target for the prevention of ASCVD. (i.e., towards the objective of minimizing any residual risk for ASCVD, in the current scenario), where the validity of control of the serum LDL-C, as one of the strongest risk factors for ASCVD, has become relatively well established.

To investigate the association of the TG/HDL-C ratio with the risk of development of $\mathrm{CAD}$, prospective cohort studies have demonstrated that a higher TG/HDL-C ratio is associated with poorer CAD-related prognosis (9). Regarding the underlying mechanisms, it has been suggested that a higher TG/HDL-C ratio is associated not only with downsizing of LDL particle size and consequent progression of atherosclerosis, but also with worsening insulin resistance and increased visceral fat deposition.

High TG is liable to be complicated by low HDL-C, and although both of these parameters are mutually independent ASCVD risk factors, the most relevant cases are those with concurrent high TG and low HDL-C, which has with a potent atherogenic effect.

Future studies should evaluate the role of other risk factors (i.e., LDL-C, hypertension, diabetes mellitus, chronic kidney disease, and obesity) and establish a concrete numerical value by which the TG/HDL-C ratio may be used as an indicator of the risk of ASCVD.

However, the TG/HDL-C ratio may prove useful for evaluating the results of an intervention study being conducted to verify the effect of a new fibrate agent endowed with more potent TG-lowering and HDL-C-increasing effects designed to suppress cardiovascular events more effectively than conventional fibrate drugs currently in use (10). In order to further reduce the risk of ASCVD, it may be beneficial to focus attention not only on the LDL-C serum level but also on the TG/HDL-C ratio, which is associated with LDL heterogeneity.

The author states that he has no Conflict of Interest (COI). 


\section{References}

1. Cannon CP, Blazing MA, Giugliano RP; IMPROVE-IT Investigators. Ezetimibe added to statin therapy after acute coronary syndromes. N Engl J Med 372: 2387-2397, 2015.

2. Bohula EA, Giugliano RP, Leiter LA, et al. Inflammatory and cholesterol risk in the FOURIER trial. Circulation 138: 131-140, 2018.

3. Miller M, Stone NJ, Ballantyne C, et al. American Heart Association Clinical Lipidology, Thrombosis, and Prevention Committee of the Council on Nutrition, Physical Activity, and Metabolism; Council on Arteriosclerosis, Thrombosis and Vascular Biology; Council on Cardiovascular Nursing; Council on the Kidney in Cardiovascular Disease. Triglycerides and cardiovascular disease: a scientific statement from the American Heart Association. Circulation 123: 2292-2333, 2011.

4. Ip CK, Jin DM, Gao JJ, et al. Effects of add-on lipid-modifying therapy on top of background statin treatment on major cardiovascular events: a meta-analysis of randomized controlled trials. Int $\mathbf{J}$ Cardiol 191: 138-148, 2015.

5. Yokoyama K, Tani S, Matsuo R, et al. Triglyceride/high-density lipoprotein cholesterol ratio may be associated with reduction in the low-density lipoprotein particle size: assessment of atherosclerotic cardiovascular disease risk. Heart Vessels 34: 227-236, 2019.

6. Moriyama K. The association between the triglyceride to highdensity lipoprotein cholesterol ratio and low-density lipoprotein subclasses. Intern Med 59: 2661-2669, 2020.

7. Tani S, Watanabe I, Anazawa T; Surugadai Atherosclerosis Regression Investigators. Effect of pravastatin on malondialdehydemodified low-density lipoprotein levels and coronary plaque regression as determined by three-dimensional intravascular ultrasound. Am J Cardiol 96: 1089-1094, 2005.

8. Tani S, Nagao K, Hirayama A. Association between urinary albumin excretion and low-density lipoprotein heterogeneity following treatment of type 2 diabetes patients with the dipeptidyl peptidase4 inhibitor, vildagliptin: a pilot study. Am J Cardiovasc Drugs 13: 443-450, 2013.

9. Bittner V, Johnson BD, Zineh I, et al. The triglyceride/high-density lipoprotein cholesterol ratio predicts all-cause mortality in women with suspected myocardial ischemia: a report from the Women's Ischemia Syndrome Evaluation (WISE). Am Heart J 157: 548-555, 2009.

10. Pradhan AD, Paynter NP, Everett BM, et al. Rationale and design of the pemafibrate to reduce cardiovascular outcomes by reducing triglycerides in patients with diabetes (PROMINENT) study. Am Heart J 206: 80-93, 2018.

The Internal Medicine is an Open Access journal distributed under the Creative Commons Attribution-NonCommercial-NoDerivatives 4.0 International License. To view the details of this license, please visit (https://creativecommons.org/licenses/ by-nc-nd/4.0/).

(C) 2020 The Japanese Society of Internal Medicine Intern Med 59: 2639-2640, 2020 